\title{
Magnetic resonance imaging of breast prostheses
}

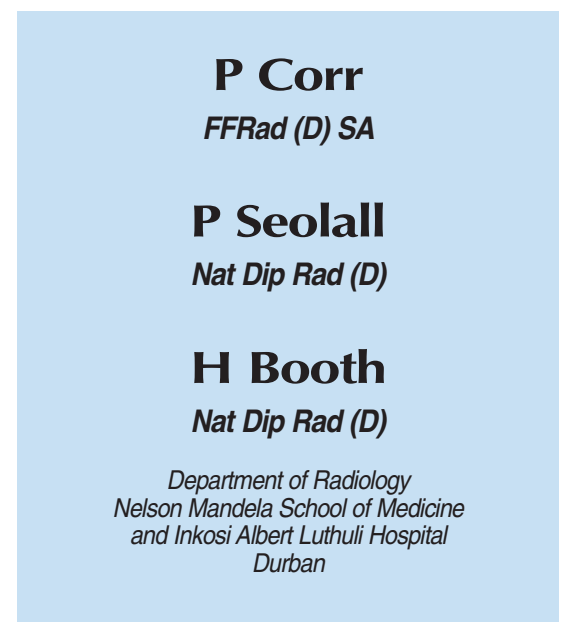

\section{Abstract}

Breast MR imaging is the most accurate imaging investigation to detect breast prosthesis rupture. Rupture is common in older prostheses ( $>10$ years post implantation) and is often asymptomatic. The radiological signs of rupture are due to collapse of the elastomer shell which is eneveloped by silicone gel and when the silicone gel separates the elastomer shell from the surrounding fibrous breast capsule.

\section{Introduction}

Breast implantation using prostheses is becoming a common plastic surgical procedure in this country. In the USA between 1 and 2 million women have had breast implantation procedures. ${ }^{1}$ The main reasons for implantation are breast reconstruction following breast cancer surgery or augmentation for cosmetic reasons. However complications following implantation of prostheses are common, occurring in $24 \%$ of patients in a series of 749 patients treated at the Mayo Clinic. ${ }^{1}$ Complications are difficult to detect by palpation, or even with ultrasound or mammographic examinations. ${ }^{2-4}$ They include rupture or leaking of silicone gel from the prosthesis and the development of silicone granulomas. Ruptured prostheses occur in $23-65 \%$ of patients and are usually silent. ${ }^{5}$ Breast MR is both sensitive and specific in detecting complications following prosthesis insertion.

In this review we illustrate the utility of breast MR imaging in detecting these complications.

\section{Breast prostheses}

Prostheses consist of a silicone-elastomer bag filled with saline, or previously with silicone gel. Currently in the USA only saline is used to fill the prostheses because of the complications associated with silicone gel rupture. ${ }^{1}$ The new generation of prostheses are manufactured with stronger and thinner capsules, so preventing rupture.

\section{Complications associ- ated with breast implantation}

Complications can be classified into those associated with the surgical procedure and those associated with the prosthesis itself. Wound complications include the development of a haematoma, seroma, wound infection, and wound dehiscence. ${ }^{6}$ Implant complications include rupture or leakage and capsular contraction by the surrounding scar fibrosis resulting in loss of the normal breast shape and consistency. ${ }^{6}$ Implant rupture or valve/port failure appears to be a function of the age of the prosthesis. ${ }^{1}$ In a large community-based prospective study of 344 patients in the USA, $55 \%$ of patients had MR evidence of rupture with silicone gel, evident outside the elastomer shell in $21 \%$ of patients. Rupture is very common 10 years after implantation. ${ }^{7}$ The prosthesis must be removed once the diagnosis of rupture is made. Capsular contraction results from a scar forming around the shell and requires re-operation in $5-20 \%$ of all patients implanted. Capsular contraction is the commonest cause of patient dissatisfaction and the commonest cause of replacement of the prosthesis.

Older prostheses contained silicone gel. Rupture of these prostheses, although asymptomatic in most patients, has been associated with collagen vascular disorders and chronic fatigue syndrome. However more current data disputes any of these associations. Currently there is debate on whether all the migrated silicone gel requires removal or not.

Detection of rupture is inaccurate with mammography and ultrasound examination. The prosthesis contents usually obscure the surrounding breast parenchyma making detection of a leak extremely difficult. MR imaging of the breast using a phased array local breast coil has been demonstrated to be the most sensitive and specific investigation to detect rupture. In large series ${ }^{7,8}$ sensitivity for ruptured prostheses was from $74 \%$ to $94 \%$, with a specificity of $85-98 \%$.

\section{Breast MR imaging signs of rupture}

Breast MR technique consists of both $\mathrm{T} 1$ and T2-weighted spin echo transverse and sagittal images, a T1-weighted transverse image with fat saturation, a T2weighted STIR sequence, and a turbo inversion recovery $\mathrm{T} 1$ transverse (TIRM) sequence with fluid suppression to detect silicone migration. The TIRM sequence suppresses fluid so making displaced silicone gel more visible. A phased array local breast coil is essential.

There are essentially two signs of rupture detectable with breast MR. The first sign is when the elastomer shell collapses and is enveloped by the silicone gel and this is detected as collapsed low-intensity lines internal to the high-intensity gel (Figs 1 and 2). The second sign is when silicone gel separates the elastometer shell from the fibrous capsule of breast tissue or the gel is found outside the shell within the breast tissue (Fig. 3). Various radiological descriptions are associated with these two signs, 


\section{REVIEW ARTICLE}

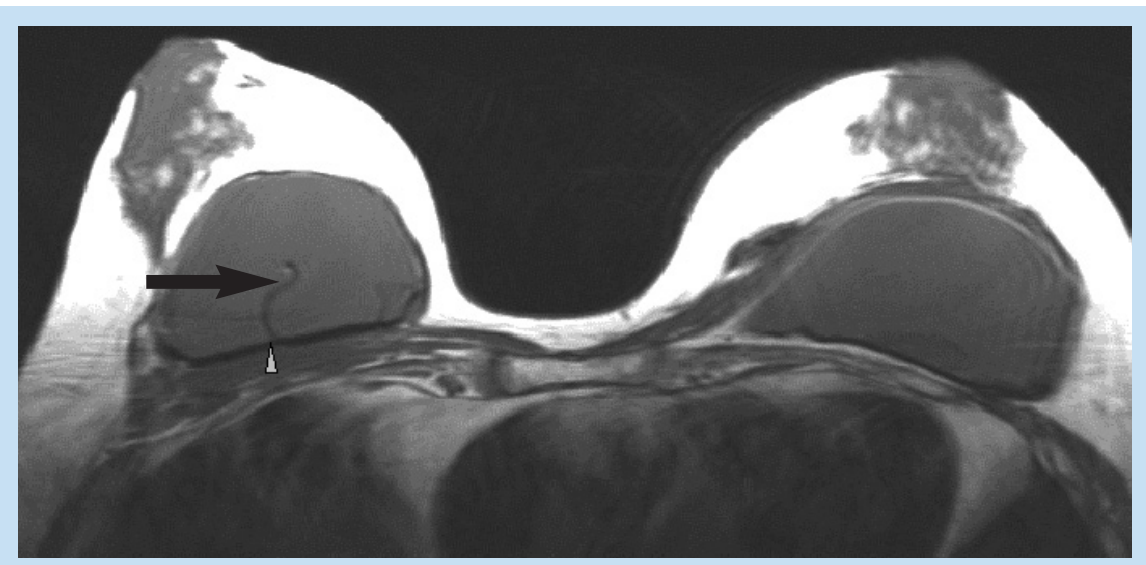

Fig. 1 T1-weighted transverse scan of a patient with implanted prostheses demonstrating early rupture of the right prosthesis with a rupture of the posterior wall of the right prosthesis ('wavy' line or 'linguine' sign) (arrow), with fluid escaping posteriorly between the elastomer shell and the breast capsule (arrowhead).

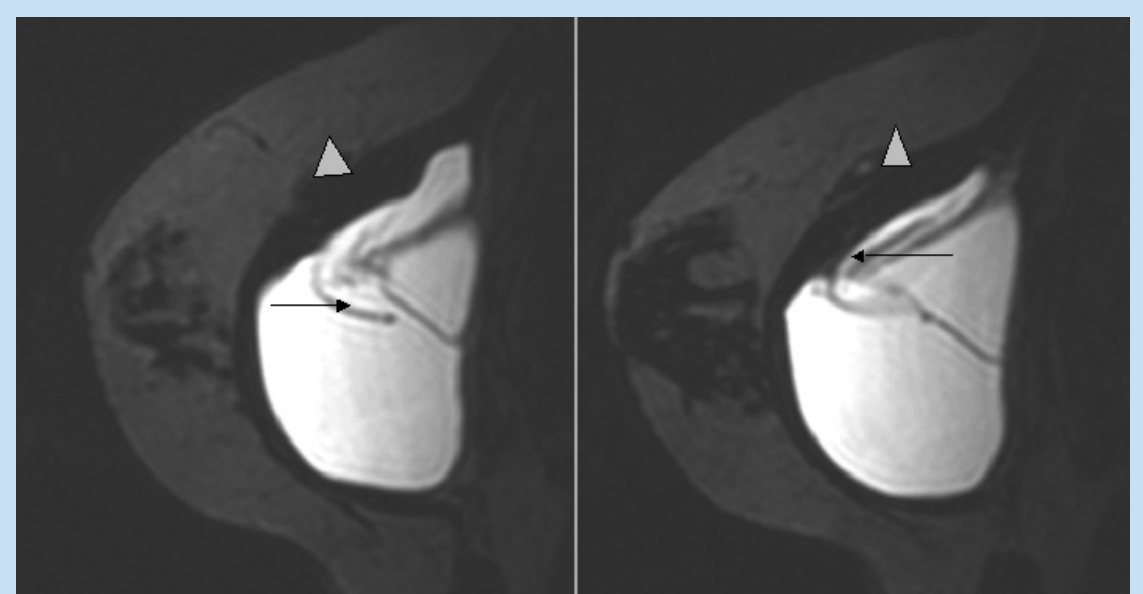

Fig. 2. T2 sagittal scan demonstrates rupture of the elastomer shell superiorly (arrow) with escape of prosthesis contents between the elastomer shell and capsule (arrowhead).

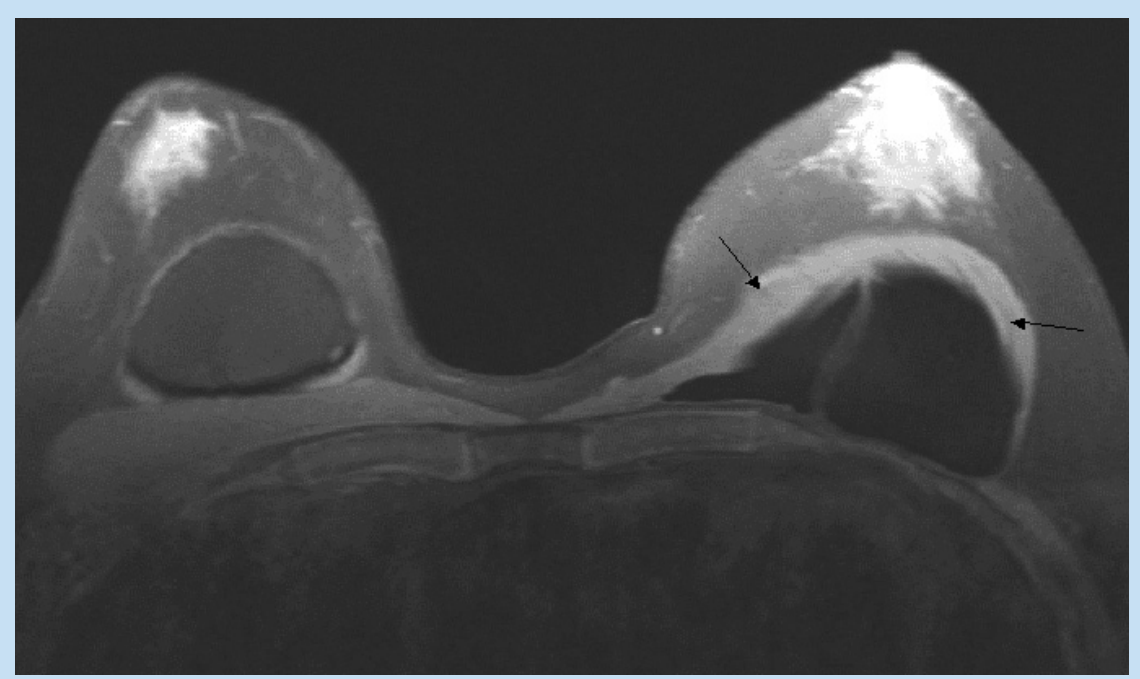

Fig.3. T2 inversion recovery magnitude or TIRM scan of a patient with a ruptured left breast prosthesis in the transverse plane. Note the prosthesis fluid collecting anterior to the breast capsule (arrows) with a rupture anteriorly of the elastomer shell. such as the linguine sign, double wavy sign, key hole sign, noose sign, and inverted tear drop sign.?

\section{Conclusions}

Breast MR imaging is a reliable and accurate investigation to detect the complication of breast prosthesis implantation. Rupture of implants is common and often asymptomatic and appears to be dependent on the age of the prosthesis.

\section{References}

1. Gabriel SE, Woods JE, O'Fallon WM, Beard CM, Kurland LT, Melton LJ. Complications leading to surgery after breast implantation $N$ Engl J Med 1997; 336: 677-682.

2. Ahn CY, DeBruhl ND, Gorczyca DP, Shaw WW, Bassett LW. Comparative silicone breast implant evaluation using mammography, sonography, and magnetic resonance imaging: experience with 59 implants. Plast Reconstr Surg 1994; 94: 620 -627.

3. Reynolds HE, Buckwalter KA, Jackson VP, Siwy BK, Alexander SG. Comparison of mammography, sonography and MR imaging in the detection of silicon implant rupture. Ann Plast Surg 1994; 33: 247-257

4. Everson LI, Parantainen H, Detlie $\mathrm{T}$, et al. Diagnosis of breast implant rupture: imaging findings and relative efficacies of imaging techniques. Am J Roentgenol 1994; 163: 57 -60.

5. Brown SL, Silverman BG, Berg WA. Rupture of silicone gel breast implants: causes, sequelae, and diagnosis. Lancet 1997; 350: 1531 -1537.

6. Mathes SJ. Breast implantation - the quest for quality and safety. N Engl J Med 1997; 336: 718719.

7. Brown SL, Middleton MS, Berg WA, Soo MS, Pennello G. Prevalence of rupture of silicone gel breast implants revealed on MR imaging in a population of women in Birmingham, Alabama Am J Roentgenol 2000; 175: 1057-1064.

8. Soo MS, Kornguth PJ, Walsh R, et al. Intracapsular implant rupture: MR findings of incomplete shell collapse. J Magn Reson Imaging 1997; 4: 724 -730. 